\title{
Controllability and Design of Unmanned Multirotor Aircraft Robust to Rotor Failure
}

\author{
Kyunam Kim*, Salar Rahili ${ }^{\dagger}$, Xichen Shi ${ }^{\ddagger}$, Soon-Jo Chung ${ }^{\S}$, and Morteza Gharib ${ }^{\mathbb{\Phi}}$. \\ California Institute of Technology, Pasadena, CA, 91125, USA
}

\begin{abstract}
A new design method for multi-rotor aircraft with distributed electric propulsion is presented to ensure a property of robustness against rotor failure from the control perspective. Based on the concept of null controllability, a quality measure is derived to evaluate and quantify the performance of a given design with the consideration of rotor failure. An optimization problem whose cost function is based on the quality measure is formulated and its optimal solution identifies a set of optimal design parameters that maximizes an aircraft's ability to control its attitude and hence its position. The effectiveness of the proposed design procedure is validated through the results of experimentation with the Autonomous Flying Ambulance model being developed at Caltech's Center for Autonomous Systems and Technologies.
\end{abstract}

\section{Introduction}

Aircraft with multiple rotors have drawn much attention in recent years because of their unique advantages such as vertical take-off and landing (VTOL) in space-constrained environments, ability to perform agile and dynamic maneuvers, and availability in a wide range of form-factors tailored to various missions. These aircraft have enabled and opened up a broad range of applications, including search and rescue [1], surveillance and monitoring [2], and transportation and delivery [3].

One important class of such aircraft is personal aerial vehicles (PAVs), or more commonly known as "flying cars." These vehicles are equipped with both distributed fan arrays and fixed wings. They are envisioned as the next generation urban transporters not only because their VTOL capability allows them to be more accessible to passengers via small landing pads in a complex urban environment than bigger aircraft that require large airports [4], but also because their wings improve energy efficiency and flight duration compared to pure rotor-based aircraft. At Caltech, we are developing an aircraft under this category as a "flying ambulance" for emergency medical services (see Fig. 11, which we believe is one of the most critical applications for such aircraft.

Clearly, safety is of the utmost importance for these aircraft and it cannot be sacrificed even when they encounter failure of some components and need to operate under abnormal conditions. Among the possible failure modes, we limit the scope of our study to rotor failure. Many multi-rotor aircraft are overactuated, which allows them to be robust against rotor failure up to a certain degree (Fig. 2). The key question that we are interested in is how to optimize design of multi-rotor aircraft such that they remain controllable when different combinations of rotors fail.

\section{A. Related Work}

A survey on fault-tolerant techniques for multi-rotor aircraft can be found in [9]. One way of handling the failure is to develop separate controllers to be used when rotors fail [10, 11]. With this approach, control authority in certain axis is often sacrificed in order to retain control in the rest. For example, it is well-known that a quadrotor cannot retain full controllability on its position and attitude when it loses one or more rotors as it becomes an underactuated system. In this case, controllers may decide to give up yaw authority [12] or to control only the reduced attitude [13]. In the literature, hexacopters have been a popular platform to study controllability because they seem to be robust to the failure of up to two rotors and be able to fly like quadrotors. Interestingly, however, researchers have shown that symmetric and collinear hexacopters subject to a single rotor failure are not fully controllable [14], and controllers with limited attitude controllability have been developed [11, 15].

\footnotetext{
*Postdoctoral Scholar, Department of Aerospace, 1200 E. California Blvd. MC 105-50, Pasadena, CA, USA

${ }^{\dagger}$ Postdoctoral Scholar, Department of Aerospace, 1200 E. California Blvd. MC 105-50, Pasadena, CA, USA.

Graduate Student, Department of Aerospace, 1200 E. California Blvd. MC 105-50, Pasadena, CA, USA.

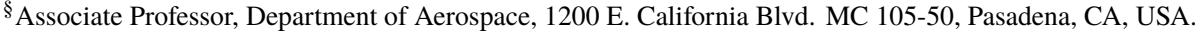

"Professor, Department of Aerospace, 1200 E. California Blvd. MC 105-50, Pasadena, CA, USA.
} 


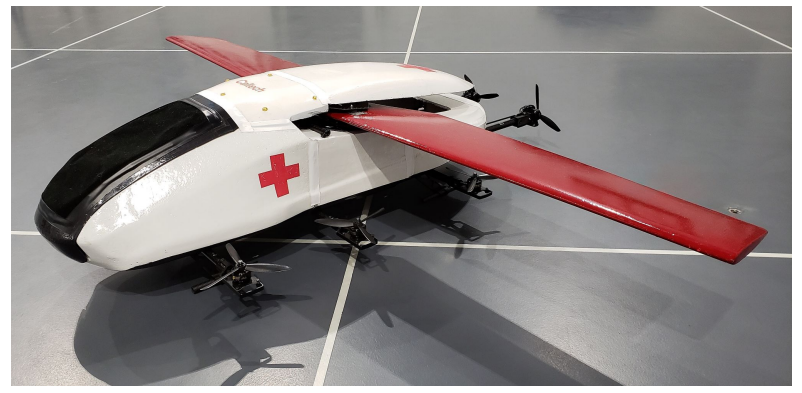

(a)

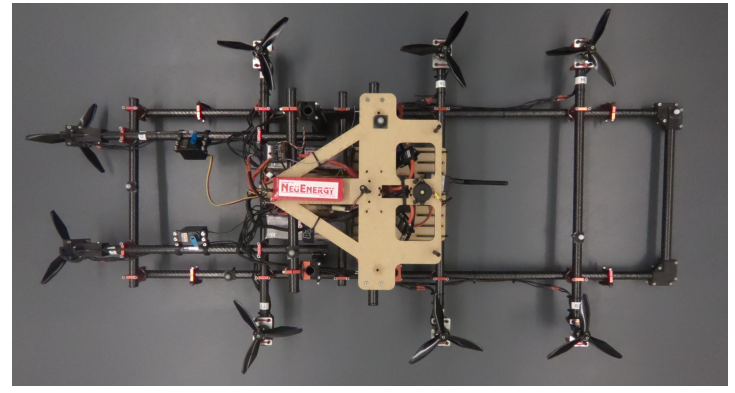

(b)

Fig. 1 (a) Autonomous Flying Ambulance being developed at Caltech's Center for Autonomous Systems and Technologies. (b) A bare-bones prototype vehicle used as a test platform.

As such, carefully designed control laws can handle certain rotor failure cases that they are intended for, but it is difficult to come up with a set of control laws that can cope with all possible cases of rotor failures. Furthermore, the resulting behavior and inherent controllability of multi-rotor aircraft are limited by their own design choices that govern the sets of generable forces and moments from the aircraft. Therefore, another, and perhaps a better, way of addressing rotor failure is to optimize the design of aircraft to be robust against rotor failure and enhance survivability of the aircraft. In this spirit, a carefully designed aircraft with only one rotor was proposed in [16], which can perform limited maneuvers and avoid unwanted crash. Hexacopters with tilted rotors were also proposed in order to retain controllability and to improve disturbance rejection after rotor failure [17-[19]. Although some asymmetric multi-rotor aircraft have been reported in the literature [20], most multi-rotor aircraft resorts to symmetric configurations [21-24], limiting exploration of the full design space as well as control performance of such aircraft.

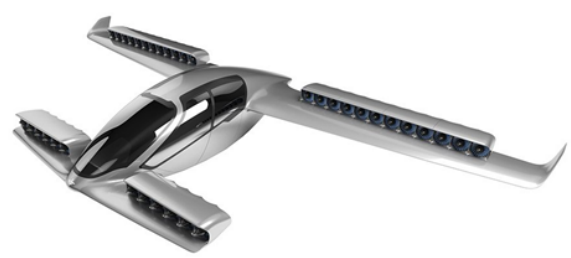

(a) Lilium, Lilium Jet [5]

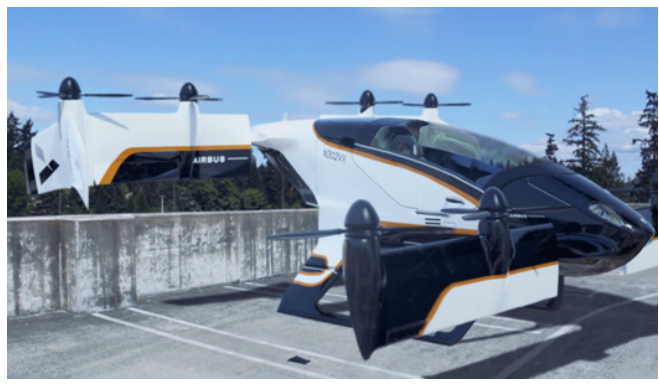

(c) Airbus, Vahana 7

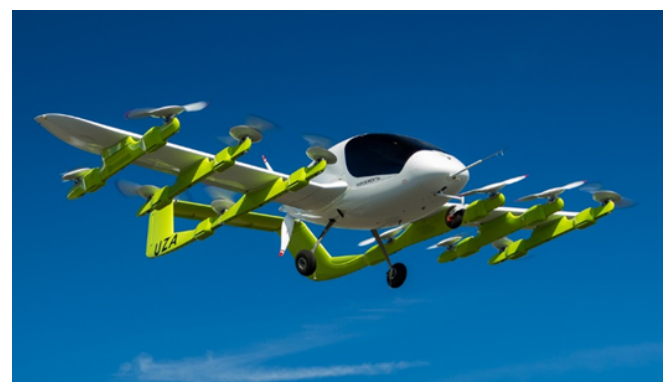

(b) Kitty Hawk, Cora 6

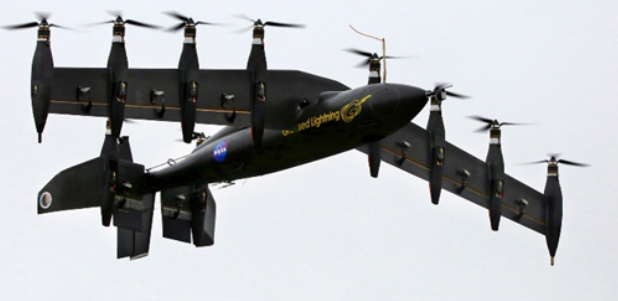

(d) NASA, GL-10 8

Fig. 2 Existing aircraft with distributed electric propulsion. 


\section{B. Contribution and Paper Organization}

In this work, we present a novel design method for multi-rotor aircraft from the control perspective, which ensures robustness against rotor failure. Specifically, we aim to optimize the design in a way that it maximizes the ability of multi-rotor aircraft to reach static hover after rotor failure, which is a desirable state once the accident happens. To this end, we first introduce multi-rotor dynamics and the notion of null controllability, and derive a condition for multi-rotor aircraft to be null controllable in Sec. II Then in Sec. III we define a quality measure based on the derived condition, which can be used to evaluate a given design with the consideration of rotor failure. Using a cost function defined based on the quality measure, we formulate an optimization problem whose solution identifies a set of optimal design parameters maximizing an aircraft's ability to control its attitude. In Sec. IV, we illustrate the design procedure with the aircraft shown in Fig. $1 \mathrm{~b}$ and provide a detailed controllability analysis of the aircraft with the optimized design. We also conduct experiment to show that the aircraft with its rotor orientations optimized can track attitude commands in all directions after two rotors fail, which is not the case when all rotors are simply vertical. For the experiment, we make use of a control allocation method recently proposed in [25], which aims to minimize energy consumption while trying to evenly distribute upward lift across all rotors to avoid potential saturation. The paper concludes in Sec. $\mathrm{V}$.

\section{Notation}

The following notations are adopted throughout this paper. $\mathbb{R}$ denotes the set of real numbers. $\mathbb{R}^{n}$ denotes the set of $n$-dimensional real vectors and $\mathbb{R}^{m \times n}$ denotes the set of $m$ by $n$ real matrices. The set of nonnegative real numbers is denoted by $\mathbb{R}_{\geq 0}$. Scalars are written in plain letters, vectors in small-bold letters, and matrices in capital-bold letters. The $i$-th element of a vector $\boldsymbol{v}$ is denoted as $v_{i} . \mathbf{0}$ is a column vector of zeros with an appropriate dimension. $\mathbf{O}$ is a zero matrix and $\mathbf{I}$ is a square identity matrix of appropriate dimensions. $\mathbf{A}=\left[a_{i j}\right]$ is a matrix with its element in $i$-th row and $j$-th column denoted as $a_{i j} . \mathbf{b}=\left[b_{i}\right]$ is a vector with its $i$-th element denoted as $b_{i} . \mathbf{K}=\operatorname{diag}\left(k_{1}, \cdots, k_{n}\right)$ is a $n$-dimensional diagonal matrix whose diagonal entries are $k_{1}, \cdots, k_{n} .\|\boldsymbol{v}\|_{2}$ is the 2-norm of $\boldsymbol{v} .\|\mathbf{A}\|_{2}$ and $\|\mathbf{A}\|_{F}$ are the 2-norm and the Frobenius norm of $\mathbf{A}$, respectively. $S_{(\cdot)}, C_{(\cdot)}$, and $T_{(\cdot)}$ denote $\sin (\cdot), \cos (\cdot)$, and $\tan (\cdot)$, respectively. The function $\max \{a, b\}$ (resp. $\min \{a, b\})$ returns $a$ if $a>b($ resp. $a<b)$ and $b$ otherwise. $\lambda_{\min }(\mathbf{B})\left(\right.$ resp. $\left.\lambda_{\max }(\mathbf{B})\right)$ denotes a minimum (resp. maximum) eigenvalue of a square matrix $\mathbf{B}$. A function whose partial derivatives exist and are continuous is in class of $C^{1} . \boldsymbol{h}_{\boldsymbol{x}}(\cdot)$ denotes the partial derivative of $\boldsymbol{h}(\cdot)$ with respect to $\boldsymbol{x}$. For a time-dependent variable $\boldsymbol{w}(t)$, its initial value at $t=t_{0}$ is denoted as $\boldsymbol{w}\left(t_{0}\right)=\boldsymbol{w}_{0}$.

\section{Controllability with Rotor Failure}

\section{A. System Dynamics}

As a precursor to the discussion of controllability, we first introduce the multi-rotor aircraft dynamics. Let us define the state vector of a multi-rotor aircraft as $\boldsymbol{x}=\left[\boldsymbol{p}^{\top}, \boldsymbol{v}^{\top}, \boldsymbol{\eta}^{\top}, \boldsymbol{\omega}^{\top}\right]^{\top}$, where $\boldsymbol{p}$ is the position of the aircraft in the inertial frame, $\boldsymbol{v}$ is the velocity of the aircraft with respect to the inertial frame expressed in the body frame, $\boldsymbol{\eta}=[\phi, \theta, \psi]^{\top}$ is a vector of ZYX Euler angles describing the orientation of the aircraft with respect to the inertial frame, and $\omega$ is the angular velocity of the aircraft with respect to the inertial frame expressed in the body frame. Furthermore, let $\mathbf{R}(\boldsymbol{\eta})$ be a rotation matrix that transforms a vector from the body frame to the inertial frame, and $\mathbf{Q}(\boldsymbol{\eta})$ be a matrix transforming angular velocities into the time derivatives of Euler angles, defined as

$$
\mathbf{R}(\boldsymbol{\eta})=\left(\begin{array}{ccc}
C_{\theta} C_{\psi} & S_{\phi} S_{\theta} C_{\psi}-C_{\phi} S_{\psi} & C_{\phi} S_{\theta} C_{\psi}+S_{\phi} S_{\psi} \\
C_{\theta} S_{\psi} & S_{\phi} S_{\theta} S_{\psi}+C_{\phi} C_{\psi} & C_{\phi} S_{\theta} S_{\psi}-S_{\phi} C_{\psi} \\
-S_{\theta} & S_{\phi} C_{\theta} & C_{\phi} C_{\theta}
\end{array}\right), \quad \mathbf{Q}(\boldsymbol{\eta})=\left(\begin{array}{ccc}
1 & S_{\phi} T_{\theta} & C_{\phi} T_{\theta} \\
0 & C_{\phi} & -S_{\phi} \\
0 & S_{\phi} / C_{\theta} & C_{\phi} / C_{\theta}
\end{array}\right)
$$

Let $\mathbf{J} \in \mathbb{R}^{3 \times 3}$ be a constant diagonal moment of inertia matrix. The aircraft dynamics are then

$$
\dot{\boldsymbol{p}}=\mathbf{R}(\boldsymbol{\eta}) \boldsymbol{v}, \quad \dot{\boldsymbol{v}}=-\boldsymbol{\omega} \times \boldsymbol{v}+\mathbf{R}^{\top}(\boldsymbol{\eta}) \boldsymbol{f}_{g}+m^{-1} \boldsymbol{f}, \quad \dot{\boldsymbol{\eta}}=\mathbf{Q}(\boldsymbol{\eta}) \boldsymbol{\omega}, \quad \mathbf{J} \dot{\boldsymbol{\omega}}=-\boldsymbol{\omega} \times \mathbf{J} \boldsymbol{\omega}+\boldsymbol{m},
$$

where $m$ is the mass, $\boldsymbol{f}_{g}$ is the gravity exerted on the aircraft expressed in the inertial frame, and $\boldsymbol{f}=\left[f_{x}, f_{y}, f_{z}\right]^{\top}$ and $\boldsymbol{m}=\left[m_{x}, m_{y}, m_{z}\right]^{\top}$ are the force and moment exerted on the aircraft by rotors, respectively. Hence, the control input to the system is $\boldsymbol{u}=\left[\boldsymbol{f}^{\top}, \boldsymbol{m}^{\top}\right]^{\top}$. 


\section{B. Static Hover}

When a multi-rotor aircraft detects rotor failure, it is desirable to steer the aircraft to static hover (i.e., $\dot{\boldsymbol{x}}=\mathbf{0}$ ) as soon as possible to cope with the emergency and land safely if necessary. If the aircraft has an ability to generate the force $\boldsymbol{f}$ and the moment $\boldsymbol{m}$ in all directions unbounded, then one can design a controller such that $\boldsymbol{f}$ and $\boldsymbol{m}$ stabilize the position and attitude dynamics, respectively. However, many practical multi-rotor aircraft have constraints on the generable $f$, and the position control often relies on the attitude control because the position dynamics in Eq. (1) is affected by attitude and angular velocities. For this reason, a control system of multi-rotor aircraft usually consists of two parts: an outer loop position controller and an inner loop attitude controller that runs faster than the position controller (Fig. 33). Under this control scheme, the position controller computes desired thrust and attitude, and the attitude controller computes desired moment based on the current and desired attitudes.

An aircraft may achieve static hover in two steps: 1) find a desired attitude $\boldsymbol{\eta}_{d}$ such that the constrained $\boldsymbol{f}$ can stabilize the position dynamics towards $\dot{\boldsymbol{p}}=\dot{\boldsymbol{v}}=\mathbf{0}$, and 2) stabilize the attitude about $\dot{\boldsymbol{\eta}}=\dot{\boldsymbol{\omega}}=\mathbf{0}$ and $\boldsymbol{\eta}=\boldsymbol{\eta}_{d}$ and $\omega=\omega_{d}=\mathbf{0}$. We make a mild assumption that the aircraft can always generate thrust large enough to counterbalance its own weight after the failure of any combination of rotors of interest. Obviously, the remaining portion of $f$ after canceling the gravity, if any, can be used for position control. Hereafter, we mainly focus on the attitude controllability of and moments exerted on multi-rotor aircraft and let $\boldsymbol{\eta}_{d}=\mathbf{0}$.

\section{Null Controllability}

Controllability analysis has been a classical problem in the controls community and many seminal works exist in the literature [26-28]. Especially for nonlinear systems, different concepts of controllability have been developed; among them, we are interested in null controllability of multi-rotor aircraft, which is related to their ability to reach static hover.

Definition 1. Consider a nonlinear system $\dot{\boldsymbol{x}}=\boldsymbol{h}(\boldsymbol{x}, \boldsymbol{u})$, where $\boldsymbol{x}(t) \in \mathbb{R}^{n}$ is a state vector, $\boldsymbol{u}(t) \in \Omega \subset \mathbb{R}^{m}$ is a control input bounded by a restraint set $\Omega$, and $\boldsymbol{h}$ is in $C^{1}$. The system is locally $\Omega$-null controllable if there exists an open set $\mathcal{V} \subset \mathbb{R}^{n}$ containing the origin such that any $\boldsymbol{x}_{0} \in \mathcal{V}$ at time $t_{0}$ can be controlled to $\boldsymbol{x}_{f}=\mathbf{0}$ in finite time $t_{f}<\infty$ by some controller satisfying $\boldsymbol{u}(t) \in \Omega$ for all time $t \in\left[t_{0}, t_{f}\right]$. The system is globally $\Omega$-null controllable if $\mathcal{V}=\mathbb{R}^{n}$.

The following theorem and corollary from [29] provide conditions for a system to be globally $\Omega$-null controllable. We refer the readers to [29] for their proofs.

Theorem 1. Consider a system $\dot{\boldsymbol{x}}=\boldsymbol{h}(\boldsymbol{x}, \boldsymbol{u})$. Suppose there exist a scalar function $V(\boldsymbol{x}): \mathbb{R}^{n} \rightarrow \mathbb{R}$, and a vector function $U(\boldsymbol{x}): \mathbb{R}^{n} \rightarrow \mathbb{R}^{m}$ in $C^{1}$ such that

(a) $V(\boldsymbol{x})=0$ if and only if $\boldsymbol{x}=\mathbf{0}$, and $V(\boldsymbol{x})>0$ otherwise;

(b) $\lim _{\|x\| \rightarrow \infty} V(x)=+\infty$;

(c) $U(x) \subset \Omega$;

(d) $\dot{V}<0$ for $\boldsymbol{x} \neq \mathbf{0}$, and $\dot{V}=0$ for $\boldsymbol{x}=\mathbf{0}$.

Then the system is globally asymptotically stable about the origin with the controller $\boldsymbol{u}(t)=U(\boldsymbol{x}(t)) \subset \Omega$ on $0 \leq t<\infty$.

If, in addition to $(d), \dot{V} \leq-\alpha V$ holds for some $\alpha>0$, then the system is exponentially stable.

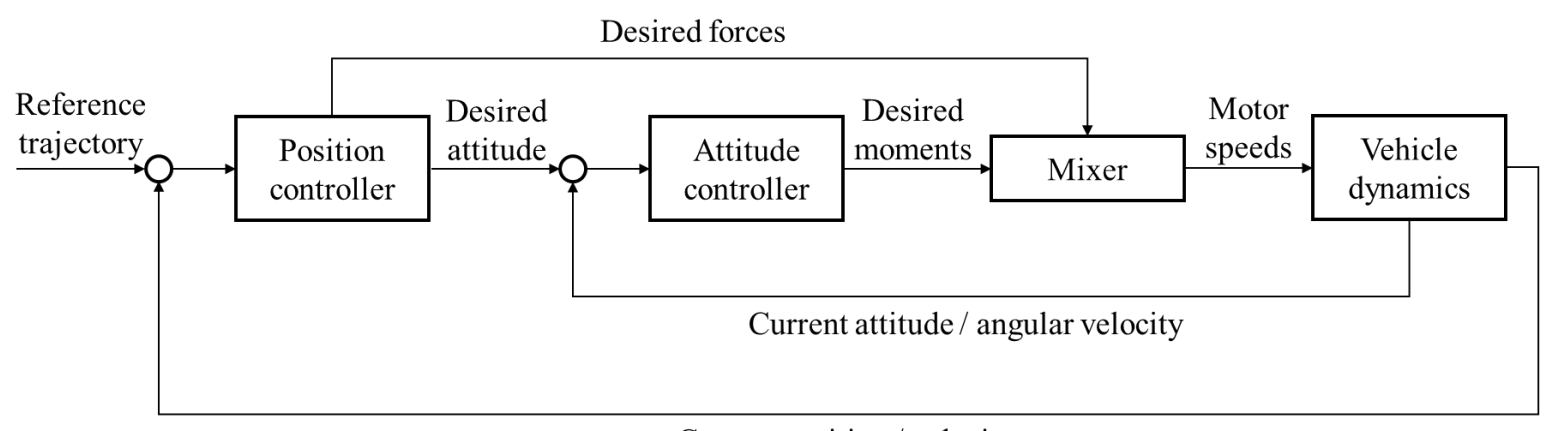

Current position / velocity

Fig. 3 A control system architecture of multi-rotor aircraft. 
Corollary 1. Consider the system in Theorem 1 and assume $V(\boldsymbol{x})$ and $U(\boldsymbol{x})$ exist satisfying the conditions therein. If the followings also hold,

(e) $\boldsymbol{h}(\mathbf{0}, \mathbf{0})=\mathbf{0}$;

(f) $\boldsymbol{u}=\mathbf{0}$ is in the interior of $\Omega$;

(g) $\operatorname{rank}\left[\mathbf{B}, \mathbf{A B}, \cdots, \mathbf{A}^{n-1} \mathbf{B}\right]=n$, where $\mathbf{A}=\boldsymbol{h}_{\boldsymbol{x}}(\mathbf{0}, \mathbf{0})$ and $\mathbf{B}=\boldsymbol{h}_{\boldsymbol{u}}(\mathbf{0}, \mathbf{0})$, then the domain of null controllability for the system is $\mathcal{V}=\mathbb{R}^{n}$.

Define the attitude state $\boldsymbol{y}=\left[\boldsymbol{\eta}^{\top}, \boldsymbol{\omega}^{\top}\right]^{\top}$. For static hover, we want to arrive at $\boldsymbol{y}\left(t_{f}\right)=\dot{\boldsymbol{y}}\left(t_{f}\right)=\mathbf{0}$ in finite time $t_{f}<\infty$ from a given initial condition at time $t_{0}$ by some controller $\boldsymbol{m}$ bounded by a control input set $\mathcal{M}$ for all $t \in\left[t_{0}, t_{f}\right]$. In other words, we want the aircraft to be $\mathcal{M}$-null controllable. Let us denote the attitude dynamics in Eq. (1) written in terms of $\boldsymbol{y}$ as $\dot{\boldsymbol{y}}=\boldsymbol{g}(\boldsymbol{y}, \boldsymbol{m})$, and let $\boldsymbol{y}$ be the domain of $\boldsymbol{y}$ where the Euler angles are valid and $\boldsymbol{\omega} \in \mathbb{R}^{3}$.

Corollary 2. The system $\dot{\boldsymbol{y}}=\boldsymbol{g}(\boldsymbol{y}, \boldsymbol{m})$ is locally $\mathcal{M}$-null controllable if $\boldsymbol{m}=\mathbf{0}$ is in the interior of $\mathcal{M}$.

Proof. We check the conditions presented in Theorem 1 and Corollary 1 . The local result is due to (c) because $\boldsymbol{m}(\boldsymbol{y}) \in \mathcal{M}$ is not guaranteed for all $\boldsymbol{y} \in \mathcal{Y}$ with a state feedback controller $\boldsymbol{m}$, especially when $\mathcal{M}$ is restricted due to rotor failure. However, it is possible to show that there exists a non-empty domain of null controllability. If $\mathcal{M}=\mathbb{R}^{3}$, then the system is $\mathcal{M}$-null controllable on $\mathcal{Y}$.

Define $\omega_{r}=\omega_{d}+\mathbf{Q}^{-1} \mathbf{K}_{1}\left(\eta_{d}-\eta\right)$ and consider the state feedback controller $\boldsymbol{m}=\mathbf{J} \dot{\omega}_{r}-\mathbf{J} \boldsymbol{\omega} \times \boldsymbol{\omega}-\mathbf{J K} \mathbf{K}_{2}\left(\boldsymbol{\omega}-\boldsymbol{\omega}_{r}\right)$. Here, $\mathbf{K}_{1}=\operatorname{diag}\left(k_{11}, k_{12}, k_{13}\right)$ and $\mathbf{K}_{2}=\operatorname{diag}\left(k_{21}, k_{22}, k_{23}\right)$ are constant positive definite matrices. Since $\boldsymbol{\eta}_{d}=\boldsymbol{\omega}_{d}=\mathbf{0}$ for static hover, $\omega_{r}=-\mathbf{Q}^{-1} \mathbf{K}_{1} \boldsymbol{\eta}$ and $\dot{\boldsymbol{\omega}}_{r}=-\dot{\mathbf{Q}}^{-1} \mathbf{K}_{1} \boldsymbol{\eta}-\mathbf{Q}^{-1} \mathbf{K}_{1} \dot{\boldsymbol{\eta}}$. Define $\widetilde{\boldsymbol{\omega}}=\boldsymbol{\omega}-\boldsymbol{\omega}_{r}$ and $\tilde{\boldsymbol{y}}=\left[\boldsymbol{\eta}^{\top}, \widetilde{\boldsymbol{\omega}}^{\top}\right]^{\top}$. Notice that $\tilde{\boldsymbol{y}}=\mathbf{0}$ if and only if $\boldsymbol{y}=\mathbf{0}$. Let $V=\frac{1}{2} \boldsymbol{\eta}^{\top} \boldsymbol{\eta}+\frac{1}{2} \widetilde{\boldsymbol{\omega}}^{\top} \widetilde{\boldsymbol{\omega}}=\frac{1}{2}\|\tilde{\boldsymbol{y}}\|_{2}^{2}$, then $V=0$ if and only if $\boldsymbol{y}=\mathbf{0}$ and $V>0$ when $\boldsymbol{y} \neq \mathbf{0}$, thereby satisfying (a). Furthermore, $V$ is radially unbounded and (b) is satisfied. With the above controller $\boldsymbol{m}$, it can be shown that $\dot{V}=-\tilde{\boldsymbol{y}}^{\top} \mathbf{P} \tilde{\boldsymbol{y}}$, where $\mathbf{P}=\left(\begin{array}{cc}\mathbf{K}_{1} & -\mathbf{Q} \\ \mathbf{O} & \mathbf{K}_{2}\end{array}\right)$ is a positive definite matrix. Hence, $\dot{V}=0$ if and only if $\boldsymbol{y}=\mathbf{0}$ and $\dot{V}<0$ otherwise, satisfying (d). In fact, $\dot{V} \leq-\lambda_{\min }(\mathbf{P})\|\tilde{\boldsymbol{y}}\|_{2}^{2}=-2 c_{1} V$ with $c_{1}=\lambda_{\min }(\mathbf{P})=\min \left\{\lambda_{\min }\left(\mathbf{K}_{1}\right), \lambda_{\min }\left(\mathbf{K}_{2}\right)\right\}>0$, and $V(\tilde{\boldsymbol{y}}) \leq e^{-2 c_{1} t} V\left(\tilde{\boldsymbol{y}}_{0}\right)$. Therefore, the closed loop system is exponentially stable and $\|\boldsymbol{\eta}\|_{2} \leq\|\tilde{\boldsymbol{y}}\|_{2} \leq e^{-c_{1} t}\left\|\tilde{\boldsymbol{y}}_{0}\right\|_{2}$.

Regarding (c), we show that there exists a non-empty subset $\mathcal{U}$ of the domain of null controllability, which guarantees $\boldsymbol{m}$ to be within a subset of $\mathcal{M}$ for all time. Define $\mathcal{B}_{\epsilon}=\left\{\boldsymbol{m} \in \mathbb{R}^{3} \mid\|\boldsymbol{m}\|_{2} \leq \epsilon\right\}$ and let $\bar{\epsilon}>0$ be the maximum radius possible within $\mathcal{M}$. Such an $\bar{\epsilon}$ always exist because the origin is in the interior of $\mathcal{M}$. We show that $\epsilon$ is determined by an initial state, and $\mathcal{U}$ is a set of $\boldsymbol{y}_{0}$ that satisfies $\epsilon\left(\boldsymbol{y}_{0}\right) \leq \bar{\epsilon}$. For this, notice $\|\boldsymbol{m}\|_{2} \leq\left\|\mathbf{J} \dot{\boldsymbol{\omega}}_{r}\right\|_{2}+\|\mathbf{J} \boldsymbol{\omega}\|_{2}\|\boldsymbol{\omega}\|_{2}+\left\|\mathbf{J} \mathbf{K}_{2} \widetilde{\boldsymbol{\omega}}\right\|_{2} \leq$ $\lambda_{\max }(\mathbf{J})\left\|\dot{\boldsymbol{\omega}}_{r}\right\|_{2}+\lambda_{\max }(\mathbf{J})\|\boldsymbol{\omega}\|_{2}^{2}+\lambda_{\max }\left(\mathbf{J K}_{2}\right)\|\widetilde{\boldsymbol{\omega}}\|_{2}$. With the controller $\boldsymbol{m}$, the closed loop attitude dynamics become $\mathbf{J} \dot{\tilde{\omega}}+\mathbf{J K}_{2} \widetilde{\boldsymbol{\omega}}=\mathbf{0}$ whose solution is $\widetilde{\boldsymbol{\omega}}=e^{-\mathbf{K}_{2} t} \widetilde{\boldsymbol{\omega}}_{0}$, and thus, $\|\widetilde{\boldsymbol{\omega}}\|_{2} \leq e^{-c_{2} t}\left\|\widetilde{\boldsymbol{\omega}}_{0}\right\|_{2}$ with $c_{2}=\lambda_{\min }\left(\mathbf{K}_{2}\right) \geq c_{1}>0$. Furthermore, $\|\boldsymbol{\omega}\|_{2} \leq\|\widetilde{\boldsymbol{\omega}}\|_{2}+\left\|\boldsymbol{\omega}_{r}\right\|_{2} \leq\|\widetilde{\boldsymbol{\omega}}\|_{2}+c_{3}\|\boldsymbol{\eta}\|_{2} \leq e^{-c_{2} t}\left\|\widetilde{\boldsymbol{\omega}}_{0}\right\|_{2}+c_{3} e^{-c_{1} t}\left\|\tilde{\boldsymbol{y}}_{0}\right\|_{2}$ with $c_{3}=\sqrt{2} \lambda_{\max }\left(\mathbf{K}_{1}\right)>0$. Also, $\left\|\dot{\boldsymbol{\omega}}_{r}\right\|_{2} \leq\left\|\dot{\mathbf{Q}}^{-1} \mathbf{K}_{1} \boldsymbol{\eta}\right\|_{2}+\left\|\mathbf{Q}^{-1} \mathbf{K}_{1} \dot{\boldsymbol{\eta}}\right\|_{2} \leq \lambda_{\max }\left(\mathbf{K}_{1}\right)\left(\|\boldsymbol{\eta}\|_{2}+\sqrt{2}\right)\|\dot{\boldsymbol{\eta}}\|_{2} \leq \lambda_{\max }\left(\mathbf{K}_{1}\right) \lambda_{\max }(\mathbf{Q})\left(\|\boldsymbol{\eta}\|_{2}+\sqrt{2}\right)\|\boldsymbol{\omega}\|_{2} \leq$ $\lambda_{\max }\left(\mathbf{K}_{1}\right) \lambda_{\max }(\mathbf{Q})\left(e^{-c_{1} t}\left\|\tilde{\boldsymbol{y}}_{0}\right\|_{2}+\sqrt{2}\right)\left(e^{-c_{2} t}\left\|\widetilde{\boldsymbol{\omega}}_{0}\right\|_{2}+c_{3} e^{-c_{1} t}\left\|\tilde{\boldsymbol{y}}_{0}\right\|_{2}\right)$. Consequently, $\|\boldsymbol{m}\|_{2}$ is upper bounded by the sum of terms that are in turn upper bounded by the terms that are exponentially decaying from the norms of initial states; we let this final upper bound of $\|\boldsymbol{m}\|_{2}$ be $\epsilon$. Therefore, for the initial states that result in $\epsilon \leq \bar{\epsilon}, \boldsymbol{m} \in \mathcal{B}_{\epsilon} \subset \mathcal{B}_{\bar{\epsilon}} \subset \mathcal{M}$ for all time and (c) is satisfied. Since $\epsilon>0$ always exist, such initial states also exist, and $\mathcal{U}$ is not empty. Furthermore, $\mathcal{U}$ is larger with a greater $\bar{\epsilon}$.

The remaining conditions are straightforward. (e) is checked by noting that $\mathbf{g}(\mathbf{0}, \mathbf{0})=\mathbf{0}$, and (f) is satisfied by the assumption. The controllability matrix constructed with $\mathbf{A}=\boldsymbol{g}_{\boldsymbol{y}}(\mathbf{0 , 0})=\left(\begin{array}{cc}\mathbf{O} & \mathbf{I} \\ \mathbf{O} & \mathbf{O}\end{array}\right)$ and $\mathbf{B}=\boldsymbol{g}_{\boldsymbol{m}}(\mathbf{0 , 0})=\left(\begin{array}{c}\mathbf{O} \\ \mathbf{J}^{-1}\end{array}\right)$ has a full rank, thereby satisfying $(\mathrm{g})$.

According to Corollary 2, one can quickly inspect if the aircraft is null controllable when its rotors are failed by checking if the resultant moment set $\mathcal{M}$ contains a neighborhood of the origin. In the proof, we also noted that it is desirable to have a greater $\bar{\epsilon}$ that will result in larger $\mathcal{U}$. Physically, $\bar{\epsilon}$ measures the magnitude of the generable moment in the weakest direction. If the evolution of attitude dynamics is too slow due to small $\bar{\epsilon}$, the position dynamics may become undesirable or energy may be drained from power source before reaching static hover. Therefore, we want the aircraft to maintain as great $\bar{\epsilon}$ as possible after rotor failure. This may be done from the initial design stage of the aircraft using, for instance, the optimization presented in Sec. III] The aircraft may also be designed in such a way that the position and orientation of its rotors are actively changed to reshape the moment set $\mathcal{M}$ and to increase $\bar{\epsilon}$. In Sec. IV] we illustrate how rotor failure affects controllability and demonstrate how aircraft design can help preserve controllability with a concrete example. 


\section{Control-Centric Design Optimization}

\section{A. Rotor Properties}

Let us denote the force and moment developed by the $i$-th rotor as $f_{i}$ and $\boldsymbol{m}_{i}$, respectively. Define a unit vector $\hat{z}_{i}$ in the direction of the rotation axis of the $i$-th rotor with respect to the aircraft's body frame, then $f_{i}=f_{i} \hat{z}_{i}$ with the magnitude of the force given by $f_{i}=\rho c_{T_{i}} d_{i}^{4} v_{i}^{2} \geq 0$ [30]. Here, $\rho$ is air density, and $c_{T_{i}}, d_{i}$, and $v_{i}$ are the thrust coefficient, diameter, and speed of the $i$-th rotor, respectively. A physical rotor has an upper limit $\bar{v}_{i}$ on its maximum rotation speed, and at this speed, it can generate a maximum thrust of $\bar{f}_{i}$. Let us also denote the largest force vector as $\bar{f}_{i}=\bar{f}_{i} \hat{z}_{i}$.

If $\boldsymbol{r}_{i}$ is the position of the $i$-th rotor with respect to the body frame, which is assumed to have its origin at the aircraft's center of gravity, then $\boldsymbol{m}_{i}=\boldsymbol{r}_{i} \times \boldsymbol{f}_{i}+\boldsymbol{\tau}_{i}$, where $\boldsymbol{\tau}_{i}=\operatorname{sgn}(i) \tau_{i} \hat{z}_{i}$ is the reaction moment exerted on the aircraft by the $i$-th rotor and $\operatorname{sgn}(i)$ is +1 or -1 depending on the rotation direction of the rotor. The magnitude of the reaction moment is $\tau_{i}=\rho c_{P_{i}} d_{i}^{5} v_{i}^{2} / 2 \pi$, where $c_{P_{i}}$ is the power coefficient. Define $\gamma_{i}=\operatorname{sgn}(i) \tau_{i} / f_{i}=\operatorname{sgn}(i) c_{P_{i}} d_{i} / 2 \pi c_{T_{i}}$, then $\boldsymbol{\tau}_{i}=\gamma_{i} \boldsymbol{f}_{i}$. As a result, $\boldsymbol{m}_{i}=f_{i} \boldsymbol{\mu}_{i}$ where $\boldsymbol{\mu}_{i}=\boldsymbol{r}_{i} \times \hat{z}_{i}+\gamma_{i} \hat{z}_{i}$. The vector $\boldsymbol{\mu}_{i}$ points in the direction of the net moment generated by the $i$-th rotor, but it is not necessarily a unit vector. Let $\overline{\boldsymbol{m}}_{i}=\bar{f}_{i} \boldsymbol{\mu}_{\boldsymbol{i}}$.

Precisely speaking, the coefficients $c_{T_{i}}$ and $c_{P_{i}}$ are functions of the rotor speed $v_{i}$; however, they are usually assumed to be constant, and this assumption has been valid in practice. We also treat these coefficients as constants in this work. Without loss of generality, we assume all rotors have the same properties to avoid unnecessary complications in the analysis: $c_{T_{i}} \equiv c_{T}, c_{P_{i}} \equiv c_{P}, d_{i} \equiv d, \bar{v}_{i} \equiv \bar{v}, \bar{f}_{i} \equiv \bar{f}$, and $\left|\gamma_{i}\right| \equiv \gamma, \forall i$.

\section{B. Moment Set and Quality Measure}

Let us denote the set of all possible moments that the rotors can exert on the aircraft as $\mathcal{M} \subset \mathbb{R}^{3}$ and define it as $\mathcal{M}=\left\{\boldsymbol{m} \in \mathbb{R}^{3} \mid \boldsymbol{m}=\sum_{i=1}^{n_{r}} a_{i} \overline{\boldsymbol{m}}_{i}, 0 \leq a_{i} \leq 1, \forall i\right\}$, where $n_{r}$ is the total number of rotors. Note that $\mathcal{M}$ is a convex set defined by the vectors $\hat{z}_{i}$ and $\boldsymbol{\mu}_{i}$, which in turn are characterized by a set of aircraft design parameters such as the total number of rotors, their locations, orientations, $c_{T}, c_{P}$, and so on. If we denote the set of all possible vectors of aircraft design parameters of interest as $\mathcal{D}$, then for each element $\boldsymbol{d} \in \mathcal{D}$, one can construct the associated set $\mathcal{M}(\boldsymbol{d})$.

To achieve our goal of design optimization, we define a quality measure $\kappa: \mathcal{D} \rightarrow \mathbb{R}_{\geq 0}$ based on some characterization of $\mathcal{M}(\boldsymbol{d})$, which enables comparison of the quality of different aircraft designs in $\mathcal{D}$. Recall from the discussion in Sec. III.C that, for null controllability, we prefer to have a large radius $\bar{\epsilon}$ of a maximal ball inscribed in $\mathcal{M}(\boldsymbol{d})$. As a further generalization of the concept, one can also consider fitting geometric objects other than a ball inside of $\mathcal{M}(\boldsymbol{d})$. For example, an ellipsoid would replace the ball if weighted control authority is desired for each dimension of $\mathcal{M}(\boldsymbol{d})$. If independence between different moment directions is desired, a rectangular cuboid may be considered. Regardless of which geometric object is chosen, we define the maximal scale of the object within $\mathcal{M}(\boldsymbol{d})$ to be our cost function $\kappa$. In all cases, the objects describe preferred moment directions and amounts in some manner, and $\kappa$ indicates the aircraft's largest ability to generate moments according to this preference. Note that the origin must be always in $\mathcal{M}(\boldsymbol{d})$ in order for $\kappa$ to be properly defined; otherwise, we let $\kappa=0$. Also, this measure corresponds to the worst case analysis in the sense that there normally exists extra control authority available beyond the geometric objects considered.

\section{Design Optimization with Rotor Failure}

We now present a design procedure to obtain an optimal $\boldsymbol{d}^{*} \in \mathcal{D}$ that results in $\mathcal{M}\left(\boldsymbol{d}^{*}\right)$ maximizing $\kappa$. Here, we consider a rectangular cuboid with a fixed ratio representing the desired relative control authority between dimensions of $\mathcal{M}(\boldsymbol{d})$, in order to ensure that certain level of control authority is independently available for each dimension of $\mathcal{M}(\boldsymbol{d})$ even after rotor failure. However, the same approach may be taken with a sphere or an ellipsoid by using the methods in [31] instead of the optimization problem formulated below.

Suppose the rectangular cuboid is defined as $C=\left\{\boldsymbol{m} \in \mathbb{R}^{3} \mid \underline{\zeta} \leq \boldsymbol{m} \leq \bar{\zeta} ; \underline{\zeta}, \bar{\zeta} \in \mathbb{R}^{3}\right\}$ with a pre-specified ratio given as $\boldsymbol{\beta}=\left[\beta_{1}, \beta_{2}, \beta_{3}\right]^{\top}$ representing the desired relative control authority for each dimension of $\mathcal{M}(\boldsymbol{d})$. Let $\kappa C$ be the scaled set of $C$ defined similar to $C$ but with lower and upper bounds of $\boldsymbol{m}$ replaced by $\kappa \underline{\zeta}$ and $\kappa \bar{\zeta}$, respectively. Note that the convex set $\mathcal{M}(\boldsymbol{d})$ is a polyhedron, which can be written as $\mathcal{M}(\boldsymbol{d})=\left\{\boldsymbol{m} \in \mathbb{R}^{3} \mid \mathbf{A} \overline{\boldsymbol{m}} \leq \mathbf{b}, \mathbf{A}=\left[a_{i j}\right] \in \mathbb{R}^{p \times 3}, \mathbf{b}=\left[b_{i}\right] \in \mathbb{R}^{p}\right\}$, where $p$ is the number of polyhedron facets. Then the following convex optimization problem solves for $\kappa^{*}$, which is the maximal scale possible while ensuring $\kappa^{*} C$ is within $\mathcal{M}(\boldsymbol{d})$ and while considering the relative importance $\beta$ : 


$$
\begin{aligned}
\kappa^{*}=\underset{\kappa}{\operatorname{argmax}} & \sum_{i=1}^{3} \log \left(\kappa\left(\bar{\zeta}_{i}-\underline{\zeta}_{i}\right)\right), \\
\text { subject to } & 0 \leq \kappa, \quad \beta_{1}^{-1}\left(\bar{\zeta}_{1}-\underline{\zeta}_{1}\right)=\beta_{2}^{-1}\left(\bar{\zeta}_{2}-\underline{\zeta}_{2}\right)=\beta_{3}{ }^{-1}\left(\bar{\zeta}_{3}-\underline{\zeta}_{3}\right), \quad \underline{\zeta}_{k}<\bar{\zeta}_{k}, k=1,2,3, \\
& a_{i j}^{+}=\max \left\{a_{i j}, 0\right\}, \quad a_{i j}^{-}=\max \left\{-a_{i j}, 0\right\}, \quad \sum_{j=1}^{3} a_{i j}^{+} \bar{\zeta}_{j}-a_{i j}^{-} \underline{\zeta}_{j} \leq b_{i}, i=1, \cdots, p .
\end{aligned}
$$

In Eq. 22], the cost function is the $\log$ of a volume of $\kappa C$. The first equality constraint enforces the relative ratio of $\kappa C$, and the last inequality constraint ensures that $\kappa C$ remains within $\mathcal{M}(\boldsymbol{d})$. The optimal set of design parameters $\boldsymbol{d}^{*}$ is then computed by solving Eq. (2) over the domain $\mathcal{D}$, that is, $\boldsymbol{d}^{*}=\operatorname{argmax}_{\boldsymbol{d} \in \mathcal{D}} \kappa^{*}$.

The above formulation, which assumed all rotors are fully functional, can be extended to handle the case with potential rotor failures as follows. Let $\mathcal{R}$ be a set whose elements are a set of rotors of which the aircraft is supposed to be robust against the failure. For each $r \in \mathcal{R}$ and $\boldsymbol{d}$, there is an associated $\mathcal{M}_{r}(\boldsymbol{d})$, where the subscript represents the loss of rotors in $r$, and also $\kappa_{r}^{*}$ obtained by solving Eq. (2) with $\mathcal{M}_{r}(\boldsymbol{d})$. Because we want to maintain at least some control authority even in the worst case scenario, we let $\kappa_{\mathcal{R}}^{*}=\min _{r \in \mathcal{R}} \kappa_{r}^{*}$ and $\boldsymbol{d}^{*}=\operatorname{argmax}_{\boldsymbol{d} \in \mathcal{D}} \kappa_{\mathcal{R}}^{*}$.

\section{Simulation and Hardware Experiment}

\section{A. Aircraft Description}

At Caltech, an aircraft that combines multiple rotors with a wing is being developed (Fig. 11). There will be two main flight modes for the aircraft; a multi-rotor mode for VTOL and slow speed flight, and a fixed-wing mode for high speed cruise flight. A bare-bones prototype of the aircraft is shown in Fig. $1 \mathrm{~b}$ and its schematic diagrams are provided in Fig. 4 To start with, some design parameters of the prototype were already chosen based on the most critical design requirements. Specifically, the prototype has a total of eight rotors around its main body, which are placed symmetric about the body longitudinal axis and are driven by electric brushless DC motors. Among them, six rotors are placed on the sides of the main body, all at the same height and equidistant from the main body to minimize aerodynamic drag when in cruise mode. The last two are located in the back, and the exact locations of the rotors are pre-determined as shown in Table 1 . Also presented in Table 2 are measured and derived rotor properties. The prototype is $112 \mathrm{~cm}$ in length, $56 \mathrm{~cm}$ in width, and $16 \mathrm{~cm}$ in height with the mass of $3.65 \mathrm{~kg}$.

The prototype will later be integrated with an aerodynamic wing and control surfaces to enable energy efficient cruise flight, and in anticipation of this, two servo motors are added to the two rear rotors to achieve thrust vectoring through tilting of the entire drive mechanisms. This will allow the prototype to gain forward acceleration without pitching the main body too much until it flies fast enough for the wing to be effective. However, this active tilting is not exploited here since we are concerned with the optimization of fixed design parameters only.

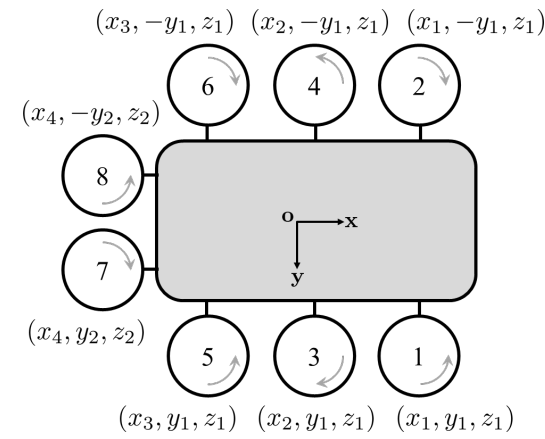

(a) Top

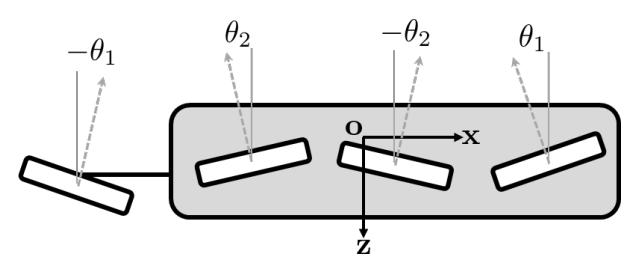

(b) Side

Fig. 4 Schematic diagrams of the prototype aircraft in Fig. $1 \mathrm{~b}$ denoted with design parameters and rotor numbers. The origin of the body frame coincides with the center of gravity. Rotor positions and orientations are defined with respect to the body frame. 
Table 1 Prototype design parameters

\begin{tabular}{c|cccccccccc}
\hline Parameter & $x_{1}$ & $x_{2}$ & $x_{3}$ & $x_{4}$ & $y_{1}$ & $y_{2}$ & $z_{1}$ & $z_{2}$ & $\theta_{1}^{*}$ & $\theta_{2}^{*}$ \\
\hline Value & $44 \mathrm{~cm}$ & $18 \mathrm{~cm}$ & $-14 \mathrm{~cm}$ & $-46 \mathrm{~cm}$ & $25 \mathrm{~cm}$ & $10 \mathrm{~cm}$ & $0 \mathrm{~cm}$ & $7 \mathrm{~cm}$ & $19^{\circ}$ & $13^{\circ}$ \\
\hline
\end{tabular}

Table 2 Rotor constants

\begin{tabular}{c|ccccccc}
\hline Constant & $c_{T}$ & $c_{P}$ & $d$ & $\bar{v}$ & $\bar{f}$ & $\gamma$ & $\rho$ \\
\hline Value & 0.1753 & 0.0911 & $15.24 \mathrm{~cm}$ & $410 \mathrm{RPS}$ & $19.47 \mathrm{~N}$ & 0.0126 & $1.225 \mathrm{~kg} / \mathrm{m}^{3}$ \\
\hline
\end{tabular}

\section{B. Design Optimization}

In order to find optimal orientations of the rotors, the optimization in Sec. III.C is solved for this specific aircraft. The design parameters for the optimization are chosen as $\boldsymbol{d}=\left[\theta_{1}, \theta_{2}\right]^{\top}$, where $\pm \theta_{i}$ is a constant, body $y$-axis tilt angle of a pair of rotors on the same row (Fig. 4). The tilt directions are chosen such that the direction of the body $z$-component of yaw moment generated by tilting matches that of the reactive yaw moment from each rotor. The domain of design parameters is $\mathcal{D}=\left\{\boldsymbol{d} \mid 0^{\circ} \leq \theta_{i} \leq 20^{\circ}, i=1,2\right\}$. The set $\mathcal{R}$ consists of all possible two-rotor failure cases. Regarding $\kappa$, it is assumed that the rectangular cuboid is centered at the origin, implying that an equal amount of control authority is desired in both positive and negative directions for each dimension of $\mathcal{M}(\boldsymbol{d})$. As a result, additional constraints are added to Eq. (2): $\underline{\zeta}_{i}=-\bar{\zeta}_{i}, \bar{\zeta}_{i}>0, i=1,2,3$. Furthermore, we assume that the ability to control roll, pitch, and yaw is of identical importance and let $\beta_{1}=\beta_{2}=\beta_{3}=1$. The optimal cost $\kappa_{\mathcal{R}}^{*}$ on $\mathcal{D}$ is depicted in Fig. 5. The optimal solution $\boldsymbol{d}^{*}=\left[\theta_{1}^{*}, \theta_{2}^{*}\right]^{\top}$ is listed in Table 1 . When there is no rotor failure, the loss in maximum vertical thrust in the body $z$-direction due to the tilt angles is relatively small; only $4 \%$ loss compared to the case of all rotors vertical.

\section{Comparison of Controllability with Rotor Failure}

A comparison between the optimized design $\boldsymbol{d}^{*}$ and a typical design choice of $\boldsymbol{d}_{0}=[0,0]^{\top}$ over the fail set $\mathcal{R}$ is shown in Table 3 . One can see that $\kappa_{r}^{*}\left(\boldsymbol{d}^{*}\right)>\kappa_{r}^{*}\left(\boldsymbol{d}_{0}\right)$ for all $r \in \mathcal{R}$. Two notable cases are $r=(3,7)$ and $r=(4,8)$, where the optimal costs are zero for $\boldsymbol{d}_{0}$. In these cases, $\boldsymbol{m}=\mathbf{0}$ is on the boundary of $\mathcal{M}_{r}\left(\boldsymbol{d}_{0}\right)$, and yaw moment is coupled with roll moment (Fig. 6a). For instance, it is not possible to generate negative yaw moment and negative roll moment at the same time for the case of $r=(3,7)$. Indeed, the linear analysis shows that these cases are not controllable around static hover [32]. In such cases, one may give up yaw authority and focus on roll control, as is commonly done in the literature, since roll directly affects position control. However, this may not be a desirable behavior for some multi-rotor aircraft, such as PAVs introduced earlier. This difficulty comes from the fact that the two failed rotors have the same rotation direction, and the aircraft suddenly loses a large amount of its capability to generate yaw moment in one direction when they fail simultaneously. Furthermore, these rotors are located on the same side with respect to the body $x$-axis, and the failure of these rotors significantly reduces the amount of generable roll moment in one direction.

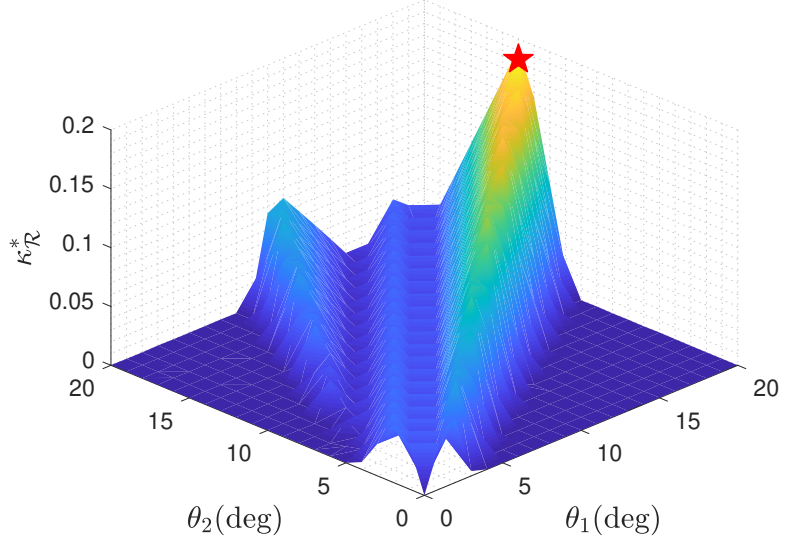

Fig. 5 Optimal cost $\kappa_{\mathcal{R}}^{*}$ on $\mathcal{D}$. The optimal design parameters are marked with a red star. 
Table 3 Comparison of $\kappa_{r}^{*}\left(d^{*}\right)$ and $\kappa_{r}^{*}\left(d_{0}\right)$ over $\mathcal{R}$

\begin{tabular}{c|cccccccccccccc}
\hline$r \in \mathcal{R}$ & $(1,2)$ & $(1,3)$ & $(1,4)$ & $(1,5)$ & $(1,6)$ & $(1,7)$ & $(1,8)$ & $(2,3)$ & $(2,4)$ & $(2,5)$ & $(2,6)$ & $(2,7)$ & $(2,8)$ & $(3,4)$ \\
\hline$\kappa_{r}^{*}\left(\boldsymbol{d}^{*}\right)$ & 2.04 & 1.01 & 0.20 & 0.20 & 2.09 & 1.89 & 1.89 & 0.20 & 1.01 & 2.09 & 0.20 & 1.89 & 1.89 & 1.34 \\
$\kappa_{r}^{*}\left(\boldsymbol{d}_{0}\right)$ & 0.48 & 0.23 & 0.16 & 0.09 & 0.48 & 0.44 & 0.44 & 0.16 & 0.23 & 0.48 & 0.09 & 0.44 & 0.44 & 0.33 \\
\hline$r \in \mathcal{R}$ & $(3,5)$ & $(3,6)$ & $(3,7)$ & $(3,8)$ & $(4,5)$ & $(4,6)$ & $(4,7)$ & $(4,8)$ & $(5,6)$ & $(5,7)$ & $(5,8)$ & $(6,7)$ & $(6,8)$ & $(7,8)$ \\
\hline$\kappa_{r}^{*}\left(\boldsymbol{d}^{*}\right)$ & 1.12 & 1.34 & 0.19 & 1.34 & 1.34 & 1.12 & 1.34 & 0.19 & 2.58 & 1.59 & 1.08 & 1.08 & 1.59 & 1.58 \\
$\kappa_{r}^{*}\left(\boldsymbol{d}_{0}\right)$ & 0.33 & 0.33 & 0 & 0.33 & 0.33 & 0.33 & 0.33 & 0 & 0.57 & 0.41 & 0.15 & 0.15 & 0.41 & 0.40 \\
\hline
\end{tabular}

The optimized design $\boldsymbol{d}^{*}$, on the other hand, ensures that $\boldsymbol{m}=\mathbf{0}$ is in the interior of $\mathcal{M}_{r}\left(\boldsymbol{d}^{*}\right)$ and maintains null controllability of the aircraft after the failure of the same sets of rotors (Fig. 6b). In this case, tilted rotors generate additional yaw moment from the body $x$-directional forces, which compensates for the loss of yaw moment from rotor failure. As a result, the coupling between roll and yaw moments is resolved near the origin, and both moments can independently be generated in positive and negative directions, without having to give up yaw control authority.

\section{Control Allocation}

Up to this point, the main tool for our analysis has been $\mathcal{M}_{r}(d)$ that represents the full set of attainable moments when rotor failure occurs. Theoretically, an aircraft should be able to generate any $\boldsymbol{m} \in \mathcal{M}_{r}(\boldsymbol{d})$; in practice, however, the problem of how to generate $\boldsymbol{m}$ (and $\boldsymbol{f}$ ) still remains, because the raw control inputs for multi-rotor aircraft are rotor speeds rather than force and moment. Solving for the mapping between rotor speeds and force and moment is referred to as a control allocation, which is implemented in a mixer (Fig. 3).

Let us define a vector of rotor speeds $\boldsymbol{v}=\left[v_{1}^{2}, v_{2}^{2}, \cdots, v_{n_{r}}^{2}\right]^{\top}$. The mapping between $\boldsymbol{u}=\left[\boldsymbol{f}^{\top}, \boldsymbol{m}^{\top}\right]^{\top}$ and $\boldsymbol{v}$ is called a control effectiveness matrix $\mathbf{E}$, the definition of which can be inferred from the relationships discussed in Sec. III.A as $\mathbf{E}=\rho c_{T} d^{4}\left(\begin{array}{ccc}\hat{z}_{1} & \cdots & \hat{z}_{n_{r}} \\ \boldsymbol{\mu}_{1} & \cdots & \boldsymbol{\mu}_{n_{r}}\end{array}\right)$ and $\boldsymbol{u}=\mathbf{E} \boldsymbol{v}$. Overactuated multi-rotor aircraft have more number of rotors than the dimension of $\boldsymbol{u}$, and thus, the goal of control allocation is essentially to map a lower dimensional space onto a higher one. Hence, there may exist multiple solutions of $\boldsymbol{v}$ generating the same $\boldsymbol{u}$. A typical way of addressing this redundancy is to solve the following problem:

$$
\begin{array}{ll}
\boldsymbol{v}^{*}=\underset{\boldsymbol{v}}{\operatorname{argmin}} & \|\boldsymbol{v}\|_{2}, \\
\text { subject to } & \boldsymbol{u}=\mathbf{E} \boldsymbol{v}, \quad \underline{\boldsymbol{v}} \leq \boldsymbol{v} \leq \overline{\boldsymbol{v}},
\end{array}
$$

where $\underline{v}$ and $\bar{v}$ are lower and upper bounds of $v$. The optimization Eq. (3) aims to find a minimum energy solution $v^{*}$ from the feasible set of $v$ generating $\boldsymbol{u}$. The downside of this approach is that Eq. (3) is required to be solved numerically on board, and it is not ideal for real-time applications where $v$ needs to be updated as fast as hardware allows.

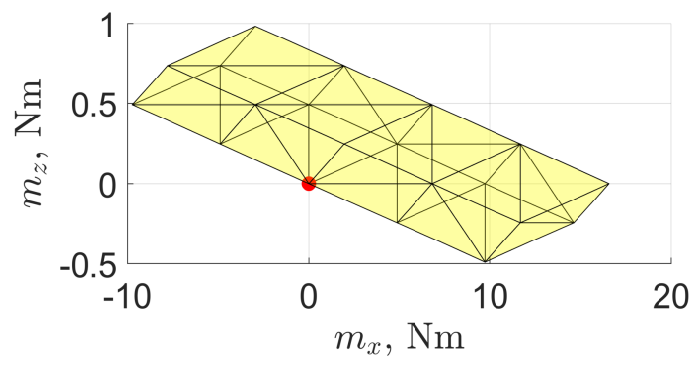

(a) $\mathcal{M}_{r}\left(\boldsymbol{d}_{0}\right), r=(3,7)$

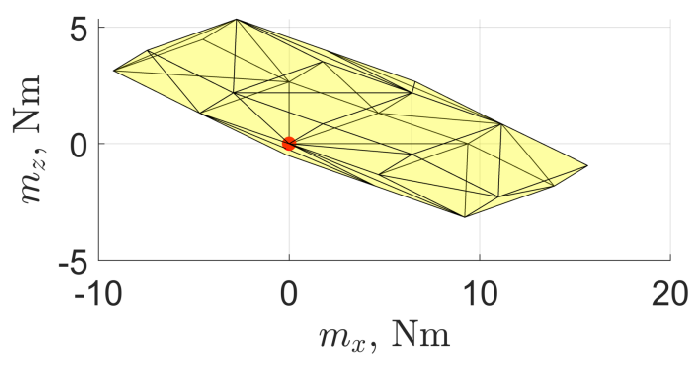

(b) $\mathcal{M}_{r}\left(\boldsymbol{d}^{*}\right), r=(3,7)$

Fig. 6 (a) $\mathcal{M}_{r}\left(d_{0}\right)$ with $r=(3,7)$. Note that the origin (marked with a red dot) is on the boundary. Because of this, generation of roll and yaw moments is coupled. (b) $\mathcal{M}_{r}\left(d^{*}\right)$ with $r=(3,7)$. Independent generation of roll and yaw moments is possible in this case and the aircraft is null controllable. The axes are not drawn in scale. 


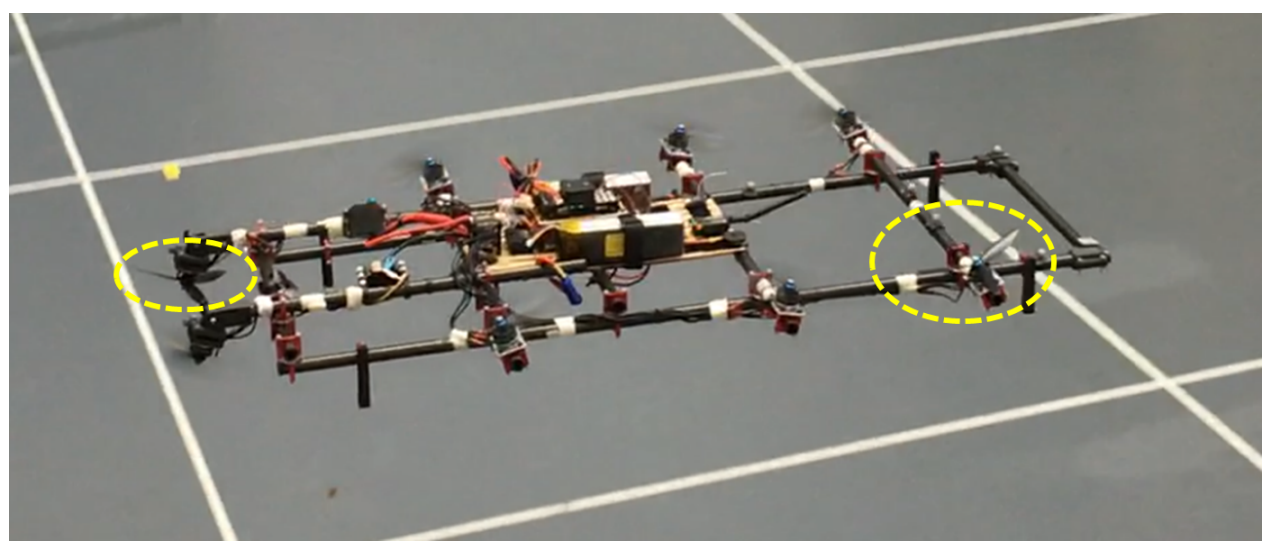

Fig. 7 The prototype in flight. The two rotors indicated by dotted circles are intentionally failed.

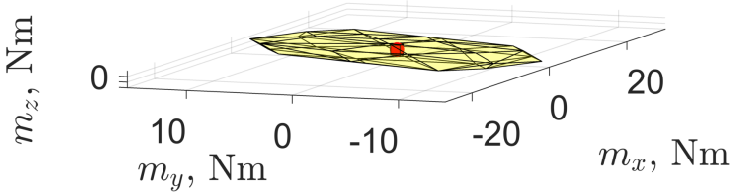

(a) $\mathcal{M}_{r}\left(\boldsymbol{d}_{0}\right), r=(1,8)$

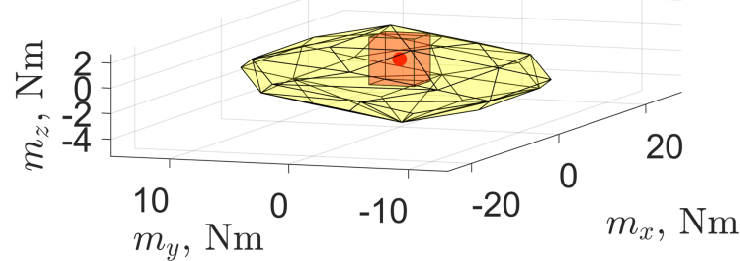

(b) $\mathcal{M}_{r}\left(\boldsymbol{d}^{*}\right), r=(1,8)$

Fig. 8 (a) $\mathcal{M}_{r}\left(d_{0}\right)$ with $r=(1,8)$. The aircraft is expected to have little yaw authority. (b) $\mathcal{M}_{r}\left(d^{*}\right)$ with $r=(1,8)$. The aircraft is expected to have a reasonable amount of roll, pitch, and yaw authority. Red boxes represent the corresponding $\kappa_{r}^{*}$.

As an alternative, the right pseudoinverse $\mathbf{E}^{+}$of $\mathbf{E}$ is usually deployed in practice, i.e., $\boldsymbol{v}^{*}=\mathbf{E}^{+} \boldsymbol{u}$. This approach can compute $v^{*}$ quickly by the use of a static mapping $\mathbf{E}^{+}$instead of solving online optimization. Indeed, this $v^{*}$ is a solution to Eq. (3) if the inequality constraint is ignored; however, $v^{*}$ may unnecessarily hit saturation when there exists another $\boldsymbol{v}$ generating $\boldsymbol{u}$ without saturation.

In the authors' previous work [25], we proposed another control allocation method that minimizes the energy consumption by optimizing the inverse $\mathbf{E}^{\prime}$ of $\mathbf{E}$ and reduces the risk of saturation by evenly distributing the effort of vertical thrust generation across all rotors:

$$
\begin{array}{ll}
\min _{\mathbf{E}^{\prime}} & \left\|\mathbf{E}^{\prime}\right\|_{F}+\frac{1}{n_{r}} \sum_{i=1}^{n_{r}}\left|e_{i}^{\prime}-\operatorname{mean}\left(\boldsymbol{e}^{\prime}\right)\right|, \\
\text { subject to } & \mathbf{E} \mathbf{E}^{\prime}=\mathbf{I}, \quad \boldsymbol{e}^{\prime} \leq \mathbf{0},
\end{array}
$$

where $\boldsymbol{e}^{\prime}$ corresponds to the column of $\mathbf{E}^{\prime}$ governing the allocation of vertical force. Note that Eq. (4) is convex and easily solved. For the experiments described in the next section, we solved this problem for $\boldsymbol{d}^{*}$ and $\boldsymbol{d}_{0}$, with and without rotor failures, and implemented the resultant $\mathbf{E}^{\prime}$ to our aircraft as static mappings for fast execution.

\section{E. Hardware Experiment}

Experiments are conducted on the prototype aircraft to test the aircraft's controllability after rotor failure. We used a Pixhawk flight controller [33] to control the prototype, with changes made to the open-source firmware to enable custom control allocation functions obtained by solving Eq. (4).

In the experiments, we intentionally failed rotors No. 1 and No. 8 of the aircraft (Fig. 7). The resulting moment sets for $\boldsymbol{d}_{0}$ and $\boldsymbol{d}^{*}$ are shown in Fig. 8. From this figure, it is anticipated that the aircraft has little yaw authority with $\boldsymbol{d}_{0}$, although it is able to generate a reasonable amount of roll and pitch moments after the rotor failure. On the other hand, the aircraft with $\boldsymbol{d}^{*}$ is expected to generate a reasonable amount of roll, pitch, and yaw moments. 

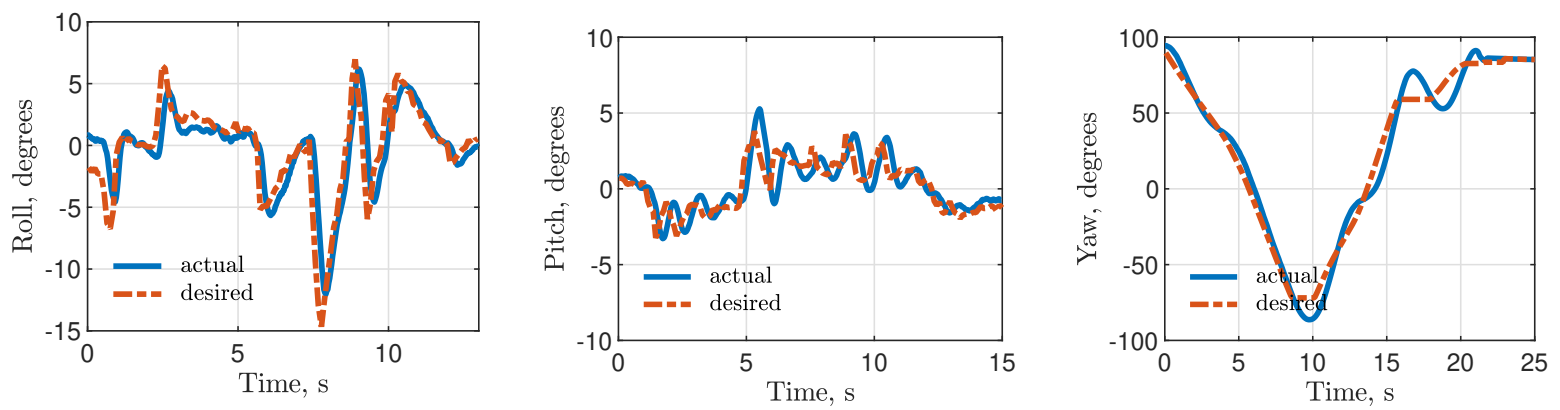

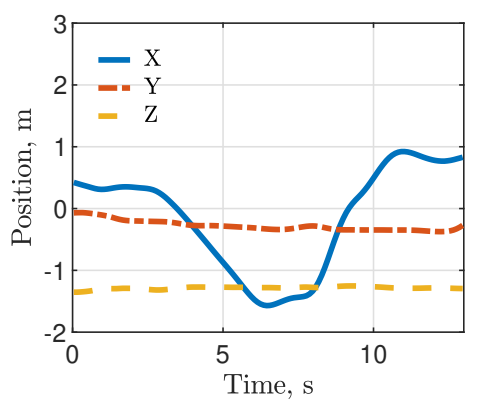

(a) Roll

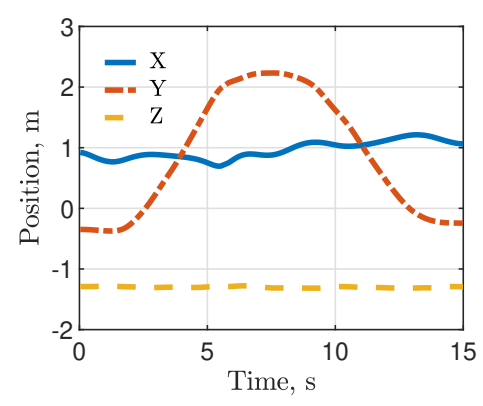

(b) Pitch

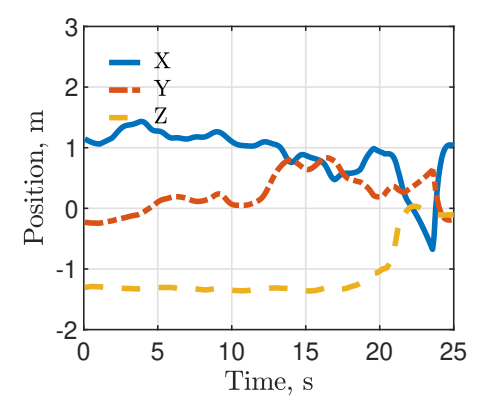

(c) Yaw

Fig. 9 Time history of angle and position states of the prototype with $d_{0}$ after perturbations were made in each axis. The prototype was able to track roll and pitch commands as well as negative yaw command. However, it started to become unstable at around $t=10 \mathrm{~s}$ in (c) while yawing in a positive direction.

In order to compare the null controllability of the prototype between $\boldsymbol{d}_{0}$ and $\boldsymbol{d}^{*}$, we configured the prototype with each of them one at a time, and then perturbed each Euler angle state of the prototype individually through manual remote control. The perturbation was made in both directions of each Euler angle to check the directional dependency of the moment generation. Then we observed the state evolution of angular states as well as the prototype's position in the inertial frame, which the body frame was initially aligned with, to see how well the prototype reaches static hover. The experiments were run without changing position and attitude controllers so that the effect of design change on the flight performance is solely verified.

The result of $\boldsymbol{d}_{0}$ is shown in Fig. 9. Figures $9 \mathrm{a}$ and $9 \mathrm{~b}$ show that the prototype was able to track roll and pitch commands and arrive at static hover once the perturbations were removed. Regarding yaw, the prototype was able to track negative yaw command while holding its position; however, yawing in positive direction caused instability to the prototype due to its limited capability to generate positive yaw moment, and the prototype was not able to hold its position anymore. This observation agrees with our discussion in Sec. IV.C. both rotors No. 1 and No. 8 are in charge of generating positive yaw moment and their failure made it difficult for the prototype to yaw in positive direction.

The result of $\boldsymbol{d}^{*}$ is shown in Fig. 10 As expected, the prototype remained null controllable after rotor failure and was able to track all of roll, pitch, and yaw commands, reaching static hover when the perturbations were removed.

\section{Conclusion}

This paper presented a novel design optimization method of multi-rotor aircraft robust to rotor failure, with the goal of maximizing a quality measure derived from the notion of null controllability that is related to the aircraft's ability to reach static hover. The design procedure was illustrated in detail with the Autonomous Flying Ambulance model being developed at Caltech's Center for Autonomous Systems and Technologies. We compared controllability of the optimized design with a design having only vertical rotors and showed that the optimized design was able to maintain null controllability for the failure cases with which the latter design was not able to do so. We also validated our results with a set of hardware experiments using the prototype aircraft. We showed that the prototype configured with the optimal design parameters can stabilize its attitude against perturbations in all angular states even after losing two rotors. 

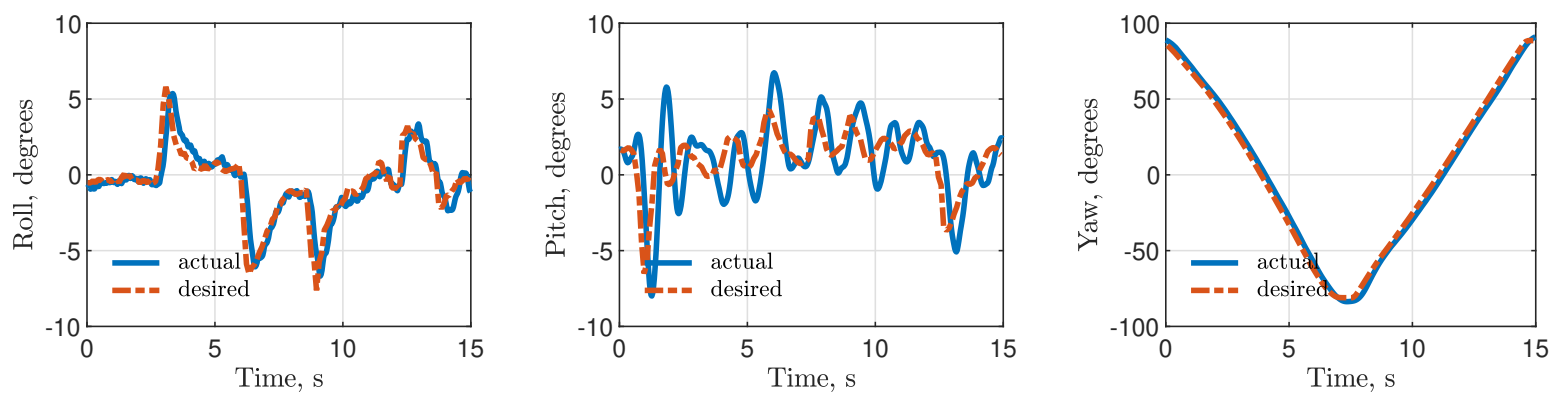

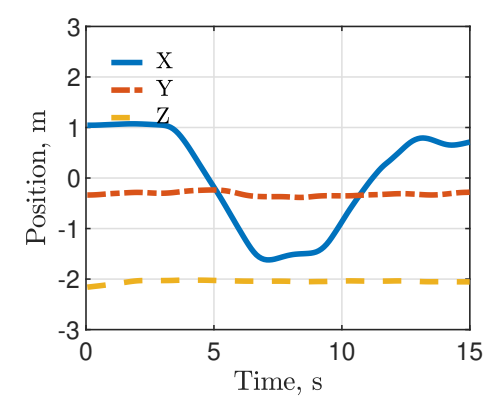

(a) Roll

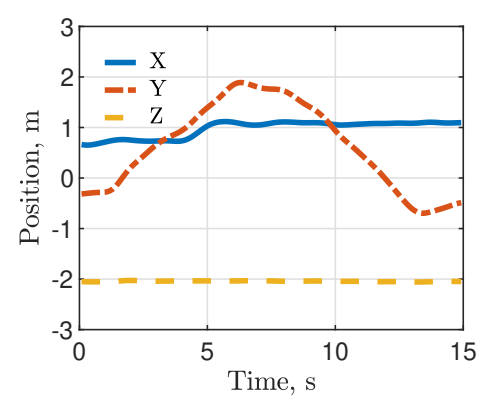

(b) Pitch

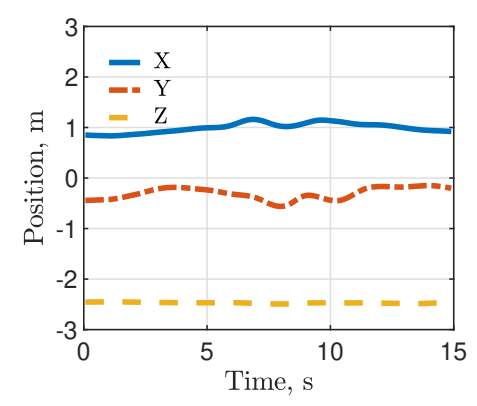

(c) Yaw

Fig. 10 Time history of angle and position states of the prototype with $\boldsymbol{d}^{*}$ after perturbations were made in each axis. The prototype was able to track roll, pitch, and yaw commands in both directions.

\section{Acknowledgment}

This work was in part funded by Caltech's Center for Autonomous Systems and Technologies and AeroVironment, Inc. The authors would like to thank Reza Nemovi and Amarbold Batzorig for their help with experiments.

\section{References}

[1] Tomic, T., Schmid, K., Lutz, P., Domel, A., Kassecker, M., Mair, E., Grixa, I. L., Ruess, F., Suppa, M., and Burschka, D., "Toward a Fully Autonomous UAV: Research Platform for Indoor and Outdoor Urban Search and Rescue," IEEE Robotics Automation Magazine, Vol. 19, No. 3, 2012, pp. 46-56.

[2] Perez, D., Maza, I., Caballero, F., Scarlatti, D., Casado, E., and Ollero, A., "A Ground Control Station for a Multi-UAV Surveillance System,” Journal of Intelligent \& Robotic Systems, Vol. 69, No. 1, 2013, pp. 119-130.

[3] Murray, C. C., and Chu, A. G., "The Flying Sidekick Traveling Salesman Problem: Optimization of Drone-Assisted Parcel Delivery," Transportation Research Part C: Emerging Technologies, Vol. 54, 2015, pp. 86 - 109.

[4] Holden, J., and Goel, N., "Fast-Forwarding to a Future of On-Demand Urban Air Transportation,” San Francisco, CA, 2016.

[5] Lilium, "Lilium Jet,"https://lilium.com/, 2018. Accessed: 2018-12-10.

[6] Kitty Hawk, "Cora,"https://cora.aero/, 2018. Accessed: 2018-12-10.

[7] Airbus, "Vahana,"https://vahana.aero/, 2018. Accessed: 2018-12-10.

[8] McSwain, R. G., Glaab, L. J., and Theodore, C. R., "Greased Lightning (GL-10) Performance Flight Research - Flight Data Report," Tech. rep., National Aeronautics and Space Administration, November 2017.

[9] Valavanis, K. P., and Vachtsevanos, G. J., Handbook of Unmanned Aerial Vehicles, Springer Publishing Company, Incorporated, 2014.

[10] Alwi, H., and Edwards, C., "Fault Tolerant Control of an Octorotor using LPV Based Sliding Mode Control Allocation," American Control Conference (ACC), 2013, pp. 6505-6510. 
[11] Falconí, G. P., and Holzapfel, F., “Adaptive Fault Tolerant Control Allocation for a Hexacopter System," American Control Conference (ACC), 2016, pp. 6760-6766.

[12] Lanzon, A., Freddi, A., and Longhi, S., "Flight Control of a Quadrotor Vehicle Subsequent to a Rotor Failure," Journal of Guidance, Control, and Dynamics, Vol. 37, No. 2, 2014, pp. 580-591.

[13] Mueller, M. W., and D’Andrea, R., "Stability and Control of a Quadrocopter Despite the Complete Loss of One, Two, or Three Propellers," Robotics and Automation (ICRA), IEEE International Conference on, 2014, pp. 45-52.

[14] Du, G.-X., Quan, Q., and Cai, K.-Y., "Controllability Analysis and Degraded Control for a Class of Hexacopters Subject to Rotor Failures,” Journal of Intelligent \& Robotic Systems, Vol. 78, No. 1, 2015, pp. 143-157.

[15] Lee, J., Choi, H. S., and Shim, H., "Fault Tolerant Control of Hexacopter for Actuator Faults using Time Delay Control Method," International Journal of Aeronautical and Space Sciences, Vol. 17, 2016, pp. 54-63.

[16] Zhang, W., Mueller, M. W., and D'Andrea, R., "A Controllable Flying Vehicle with a Single Moving Part," IEEE International Conference on Robotics and Automation (ICRA), 2016, pp. 3275-3281.

[17] Michieletto, G., Ryll, M., and Franchi, A., "Control of Statically Hoverable Multi-Rotor Aerial Vehicles and Application to Rotor-Failure Robustness for Hexarotors," IEEE International Conference on Robotics and Automation (ICRA), 2017, pp. 2747-2752.

[18] Giribet, J. I., Sanchez-Pena, R. S., and Ghersin, A. S., "Analysis and Design of a Tilted Rotor Hexacopter for Fault Tolerance," IEEE Transactions on Aerospace and Electronic Systems, Vol. 52, No. 4, 2016, pp. 1555-1567.

[19] Ryll, M., Bicego, D., and Franchi, A., "Modeling and Control of FAST-Hex: A Fully-Actuated by Synchronized-Tilting Hexarotor," IEEE/RSJ International Conference on Intelligent Robots and Systems (IROS), 2016, pp. 1689-1694.

[20] Achtelik, M., Doth, K.-M., Gurdan, D., and Stumpf, J., "Design of a Multi Rotor MAV with Regard to Efficiency, Dynamics and Redundancy," AIAA Guidance, Navigation, and Control Conference, 2012, pp. 4779-4795.

[21] Crowther, B., Lanzon, A., Maya-Gonzalez, M., and Langkamp, D., "Kinematic Analysis and Control Design for a Nonplanar Multirotor Vehicle,” Journal of Guidance, Control, and Dynamics, Vol. 34, No. 4, 2011, pp. 1157-1171.

[22] Kaufman, E., Caldwell, K., Lee, D., and Lee, T., "Design and Development of a Free-Floating Hexrotor UAV for 6-DOF Maneuvers," IEEE Aerospace Conference, 2014, pp. 1-10.

[23] Ryll, M., Bülthoff, H. H., and Giordano, P. R., "A Novel Overactuated Quadrotor Unmanned Aerial Vehicle: Modeling, Control, and Experimental Validation,” IEEE Transactions on Control Systems Technology, Vol. 23, No. 2, 2015, pp. 540-556.

[24] Efraim, H., Shapiro, A., and Weiss, G., "Quadrotor with a Dihedral Angle: On the Effects of Tilting the Rotors Inwards," Journal of Intelligent \& Robotic Systems, Vol. 80, No. 2, 2015, pp. 313-324.

[25] Shi, X., Kim, K., Rahili, S., and Chung, S.-J., "Nonlinear Control of Autonomous Flying Cars with Wings and Distributed Electric Propulsion," IEEE Conference on Decision and Control (CDC), Miami Beach, FL, 2018.

[26] Kalman, R. E., et al., "Contributions to the Theory of Optimal Control,” Bol. Soc. Mat. Mexicana, Vol. 5, No. 2, 1960, pp. $102-119$.

[27] Hermann, R., and Krener, A., "Nonlinear Controllability and Observability," IEEE Transactions on automatic control, Vol. 22, No. 5, 1977, pp. 728-740.

[28] Khalil, H. K., Nonlinear Systems, $3^{\text {rd }}$ ed., Prentice hall Upper Saddle River, NJ, 2002.

[29] Lee, E. B., and Markus, L., Foundations of Optimal Control Theory, John Wiley \& Sons, 1967.

[30] McCormick, B. W., Aerodynamics, Aeronautics, and Flight Mechanics, $2^{\text {nd }}$ ed., Wiley New York, 1995.

[31] Boyd, S., and Vandenberghe, L., Convex Optimization, Cambridge university press, 2004.

[32] Du, G.-X., Quan, Q., Yang, B., and Cai, K.-Y., “Controllability Analysis for Multirotor Helicopter Rotor Degradation and Failure," Journal of Guidance, Control, and Dynamics, Vol. 38, No. 5, 2015, pp. 978-985.

[33] Meier, L., Tanskanen, P., Heng, L., Lee, G. H., Fraundorfer, F., and Pollefeys, M., "PIXHAWK: A Micro Aerial Vehicle Design for Autonomous Flight using Onboard Computer Vision,” Autonomous Robots, Vol. 33, No. 1-2, 2012, pp. 21-39. 